AperTO - Archivio Istituzionale Open Access dell'Università di Torino

\title{
Functions of the first Baire class
}

\section{This is a pre print version of the following article:}

Original Citation:

\section{Availability:}

This version is available http://hdl.handle.net/2318/1722701

since 2020-01-13T12:41:25Z

Published version:

DOI:10.1017/bsl.2018.83

Terms of use:

Open Access

Anyone can freely access the full text of works made available as "Open Access". Works made available under a Creative Commons license can be used according to the terms and conditions of said license. Use of all other works requires consent of the right holder (author or publisher) if not exempted from copyright protection by the applicable law. 
RAPHaËl CARRoy. Functions of the first Baire class. Université Paris 7 - Denis Diderot \& Université de Lausanne. 2013. Supervised by Olivier Finkel \& Jacques Duparc. MSC: Primary 26A21, 54C05. Keywords: Continuous functions, infinite games, well-quasi-orders, continuous reducibility.

We aim at starting an analysis of definable functions similar to the Wadge theory for definable sets, focusing more specifically on Baire class 1 functions between 0-dimensional Polish spaces. To parallel Wadge's analysis, we break this study in two parts. The first concerns subclasses of the first Baire class characterisable by infinite games, while the second looks at the quasi-order of continuous reducibility on continuous functions.

Here $X, Y, X^{\prime}$ and $Y^{\prime}$ are variables for Polish 0-dimensional (P0D for short) spaces, considered as closed subspaces of the Baire space of infinite sequences of natural numbers.

Playing in the first Baire class. 1 We consider various infinite games with a function $f: X \rightarrow Y$ as parameter. The simplest one sees Player $I$ choosing a natural number $x_{i}$ and Player $I I$ a natural $y_{i}$ at the $i$-th round, thus building two infinite sequences $x$ and $y$. Player $I I$ then wins the game if and only if $f(x)=y$. In this game, Player $I I$ has a winning strategy if and only if $f$ is Lipschitz; we say that the game characterises Lipschitz functions.

New rules giving more and more power to Player $I I$ will then characterise larger and larger classes of functions. For instance, allowing Player II to skip her turn as often as she wants characterises continuous functions. These first two games were defined by Wadge in his $\mathrm{PhD}$ thesis. We consider three others.

In the backtrack game, defined by van Wesep in his $\mathrm{PhD}$ thesis, Player II can erase what she did at the previous round, but only finitely many times during a run. It characterises functions that are $\sigma$-continuous with closed witnesses.

Duparc in his PhD thesis defined the eraser game by allowing Player II to erase infinitely often. This one characterises Baire class 1 functions.

The third one is a refinement of the first one, defined by Motto Ros in his $\mathrm{PhD}$ thesis. It is called the $\alpha$-bounded backtrack game, because the erasing ability of $I I$ is bounded by some countable ordinal $\alpha$.

We prove that all three games are determined. Observe that instead of considering only Borel functions and use Martin's result on Borel determinacy, we show a stronger determinacy result.

Theorem 1. For all functions $f: X \rightarrow Y$, the eraser game, the backtrack game and the $\alpha$-bounded backtrack game with parameter $f$ are determined.

We in fact define the finer notion of an aggressive strategy for Player $I$ to get a stronger theorem; namely if $I I$ has no winning strategy in the backtrack game with parameter $f: X \rightarrow Y$, then $I$ has an aggressive winning strategy.

As a corollary, we give a new proof, purely game-theoretic, of the Baire Lemma on pointwise convergence.

\footnotetext{
${ }^{1}$ This was published in Mathematical Logic Quarterly, vol. 60, n. 1-2, pp. 118-132, 2014
} 
A quasi-order for continuous functions. 2 Given two functions $f: X \rightarrow$ $Y$ and $g: X^{\prime} \rightarrow Y^{\prime}$, we write $f \leq g$ and say that $g$ continuously reduces $f$ if there are two continuous functions $\sigma: X \rightarrow X^{\prime}$ and $\tau: \operatorname{Im}(g \circ \sigma) \rightarrow Y$ satisfying $f=\tau \circ g \circ \sigma$. We write $f \equiv g$ when both $f \leq g$ and $g \leq f$ hold.

Computable versions of this quasi-order, introduced by Weihrauch, have become central in computable analysis.

We first prove that any two continuous functions $f: X \rightarrow Y$ and $g: X^{\prime} \rightarrow Y^{\prime}$ with uncountable ranges are continuously bi-reducible. We then study more specifically functions with countable image; we let both $C_{\infty}$ denote the class of all continuous functions between P0D spaces and $C$ denote the subclass of $C_{\infty}$ of functions with countable range.

We define the Cantor-Bendixson rank $\mathrm{CB}(f)$ of a function $f$ in $\mathrm{C}$. This rank generalises the usual Cantor-Bendixson rank on closed sets, in the sense that $\mathrm{CB}\left(\operatorname{Id}_{F}\right)=\mathrm{CB}(F)$ for all closed $F$. This rank stratifies $\mathrm{C}$ in classes $\mathrm{C}_{\alpha}$ of all functions in $\mathrm{C}$ of Cantor-Bendixson rank $\alpha$, for $\alpha$ countable.

We isolate two infinitary operations on P0D spaces, called the gluing and the pointed gluing. We give universal properties for these, and prove that they generate, from the empty set, all countable P0D spaces up to homeomorphism.

We then define the gluing and pointed gluing on sequences of functions so that the operations on sets and those on functions commute with the identity functor.

Letting $C^{\star}$ be the subclass of $C$ of functions with compact domain, we prove

Theorem 2. The relation $\leq$ is a well-order on $\mathrm{C}^{\star} / \equiv$ of order type $\omega_{1}$.

Using $\mathrm{C}^{\star}$ as a leverage point in $\mathrm{C}$, we describe the general structure of $(\mathrm{C}, \leq)$.

Theorem 3. Given $f, g$ in $\mathrm{C}, \lambda<\omega_{1}$ a limit ordinal and $n$ a natural, we have

1. if $\mathrm{CB}(f)=\mathrm{CB}(g)=\lambda$ then $f \equiv g$,

2. if $\mathrm{CB}(f)=\lambda+n$ and $\mathrm{CB}(g)=\lambda+2 n+1$, then $f \leq g$.

In particular, if $\left(\mathrm{C}_{\alpha}, \leq\right)$ is a well-quasi-order (wqo) for all $\alpha \in \omega_{1}$ then $\left(C_{\infty}, \leq\right)$ is a wqo. The question of wether $C_{\infty}$ is a wqo or not remains open.

Applying Theorem 3 to the subclass of identity functions, we obtain an alternative proof that topological embeddability on P0D spaces is a wqo. This result is also obtained as a corollary of Laver's celebrated result on labelled trees.

Raphaël Carroy.

E-mail: raphael.carroy@univie.ac.at.

URL: http://www.logique.jussieu.fr/ carroy/papers/These.pdf.

${ }^{2}$ This was published in the Journal of Symbolic Logic, vol. 78, n. 2, pp. 633-648, 2013 